\title{
MORAVA $K$-THEORY AND THE FREE LOOP SPACE
}

\author{
JOHN MCCLEARY AND DENNIS A. MCLAUGHLIN
}

(Communicated by Frederick R. Cohen)

\begin{abstract}
We generalize a result of Hopkins, Kuhn, and Ravenel relating the $n$th Morava $K$-theory of the free loop space of a classifying space of a finite group to the $(n+1)$ st Morava $K$-theory of the space. We show that the analogous result holds for any Eilenberg-Mac Lane space for a finite group. We also compute the Morava $K$-theory of the free loop space of a suspension, and comment on the general problem.
\end{abstract}

It has been suspected for some time that there is a close relationship between $v_{n+1}$-periodic information of a space $M$ and the $v_{n}$-periodic information of the free loop space, $\mathscr{L} M=\operatorname{map}\left(S^{1}, M\right)$. Although the exact nature of this phenomenon is still unclear, there are several indications as to what it might be. In $[13,14]$ Witten has observed that the index of the Dirac operator on $\mathscr{L} M$ "is" the elliptic genus [11]. One concludes from this, that the Chern character in elliptic cohomology [9] should agree with the Chern character of a suitably defined equivariant $K$-theory of the free loop space $[1,11]$. On the other hand, it was shown in [7] that for the classifying space of a finite group, $B G$, one has

$$
\chi\left(K(n)_{*}(\mathscr{L} B G)\right)=\chi\left(K(n+1)_{*}(B G)\right),
$$

where $K(n)_{*}$ is the $n$th Morava $K$-theory associated to a fixed odd prime $p$ and $\chi$ is the Euler characteristic.

The purpose of this paper is to make the observation, perhaps surprising, that this relation still holds when $B G$ is replaced by any Eilenberg-Mac Lane space $K(\pi, q)$ for a finite group $\pi$. The proof is just a direct comparison and relies on the computations in [10]. We are unable to give a geometric proof of this result, though it certainly provides more evidence for the relationship considered above.

In the final section we compute $K(n)_{*}(\mathscr{L} X)$ in the case where $X$ is a suspension, and we make some remarks about the general case.

\section{Preliminaries}

In this section we recall the definition and main properties of Morava $K$ theory. The main reference is [8]. Let $p$ be an odd prime. Localizing complex

Received by the editors May 8, 1990.

1980 Mathematics Subject Classification (1985 Revision). Primary 55P35, 55N20.

$K e y$ words and phrases. Free loop space, Morava $K$-theory. 
cobordism at $p$ leads to a splitting of the representing spectrum $M U$ into summands that are suspensions of Brown-Peterson spectra $B P$ [2]. The coefficient ring for Brown-Peterson homology is given by

$$
B P_{*}=\pi_{*}(B P) \cong \mathbb{Z}_{(p)}\left[v_{1}, v_{2}, \ldots\right],
$$

where $\mathbb{Z}_{(p)}$ denotes the integers localized at the prime $p$, and the classes $v_{i}$ have dimension $2\left(p^{i}-1\right)$. By killing off the ideal $\left(p, v_{1}, \ldots, v_{n-1}, v_{n+1}, \ldots\right)$, one constructs a connective spectrum $k(n)$ satisfying $\pi_{*}(k(n)) \cong \mathbb{F}_{p}\left[v_{n}\right]$. Inverting the periodicity element $v_{n}$, we obtain a spectrum

$$
K(n)=\lim _{\rightarrow v_{n}} \Sigma^{-2\left(p^{n}-1\right)} k(n),
$$

called the $n$th Morava $K$-theory. For $n=0$ it is customary to think of $K(0)=$ $H \mathbb{Q}$, ordinary rational homology, and $K(1)$ is a summand in $\bmod p \operatorname{complex}$ $K$-theory.

The basic properties of $K(n)$ are summarized in the following:

Theorem 1.1. For an odd prime $p$ and $n>0$, the Morava $K$-theories, $K(n)_{*}(-)$, satisfy

(1) $K(n)_{*}(-)$ is a multiplicative homology theory with coefficient ring given by the graded field

$$
K(n)_{*}=\pi_{*}(K(n)) \cong \mathbb{F}_{p}\left[v_{n}, v_{n}^{-1}\right] .
$$

(2) The exterior product induces a Künneth isomorphism:

$$
K(n)_{*}(X) \otimes_{K(n)_{*}} K(n)_{*}(Y) \stackrel{\cong}{\rightrightarrows} K(n)_{*}(X \times Y) .
$$

The existence of a Künneth isomorphism is crucial to our calculations. Ordinary homology with field coefficients and the Morava $K$-theories are the only generalized homology theories enjoying this property for all spaces.

We also recall the cyclically graded version of $K(n)_{*}$. This is defined by setting

$$
\overline{K(n)_{*}}(X)=\mathbb{F}_{p} \otimes_{K(n) *} K(n)_{*}(X),
$$

where the tensor product is formed via the algebra homomorphism

$$
\mathbb{F}_{p}\left[v_{n}, v_{n}^{-1}\right] \rightarrow \mathbb{F}_{p}, \quad \text { given by } v_{n} \mapsto 1
$$

We have

$$
\overline{K(n)}_{\bar{j}}(X) \equiv K(n)_{j}(X), \quad \text { for } j \equiv \bar{j} \bmod 2\left(p^{n}-1\right),
$$

which is well defined since multiplication by $v_{n}$ is an isomorphism,

$$
K(n)_{t}(X) \cong K(n)_{t+2\left(p^{n}-1\right)}(X) .
$$

It is clear from this description that

$$
\operatorname{dim}_{K(n)_{*}} K(n)_{*}(X)=\operatorname{dim}_{\mathbb{F}_{p}}{\overline{K(n)_{*}}}_{*}(X) .
$$

\section{The Morava $K$-Theory of Eilenberg-Mac Lane spaces}

In this section we recall the computation of the Morava $K$-theory of the Eilenberg-Mac Lane spaces $K(\pi, q)$ for fixed abelian group $\pi$ [10] and use this to obtain our main result, Theorem 2.4 . 
There is a cup product pairing,

$$
K(\pi, q) \wedge K(\pi, r) \rightarrow K(\pi, q+r),
$$

which induces a product

$$
\circ: K(n)_{*}(K(\pi, q)) \otimes_{K(n)_{*}} K(n)_{*}(K(\pi, r)) \rightarrow K(n)_{*}(K(\pi, q+r)) .
$$

Along with the Hopf algebra structure already on $K(n)_{*}(K(\pi, n))$, one obtains the structure of a Hopf ring on $K(n)_{*}(K(\pi, \bullet))$ as follows.

Theorem 2.1 [10]. Let $p$ be an odd prime, and $\pi=\mathbb{Z} / p^{j} \mathbb{Z}$ for some $j \geq 1$. The Hopf ring $K(n)_{*}(K(\pi, \bullet))$ is free over $K(n)_{*}\left[\mathbb{Z} / p^{j} \mathbb{Z}\right]$ on the Hopf algebra $K(n)_{*}(K(\pi, 1))$.

The ring $K(n)_{*}\left(K\left(\mathbb{Z} / p^{j} \mathbb{Z}, 1\right)\right)$ is free over $K(n)_{*}$ on elements

$$
a_{i} \in K(n)_{2 i}(K(\pi, 1)), \quad 0 \leq i<p^{n j},
$$

with algebra structure given by a divided power algebra and the relations

$$
a_{(n+i-1)}^{* p}=v_{n}^{p^{i}} a_{(i)}, \quad \text { where } a_{(i)}=a_{p^{i}} .
$$

Using the product $\circ$, we obtain elements

$$
a_{I}=a_{\left(i_{1}\right)} \circ \cdots \circ a_{\left(i_{q}\right)}
$$

in $\left.K(n)_{*}\left(K \mathbb{Z} / p^{j} \mathbb{Z}, q\right)\right)$ for $I=\left(i_{1}, \ldots, i_{q}\right)$. Let $P_{R}[x, k]$ denote the truncated polynomial algebra $R[x] /\left(x^{p^{k}}\right)$. The following theorem summarizes the results we need:

Theorem $2.2[10,11.1]$. For $p$ an odd prime, $j \geq 1$, we have as algebras:

(1) $K(n)_{*}\left(K\left(\mathbb{Z} / p^{j} \mathbb{Z}, 0\right)\right) \cong K(n)_{*}\left[\mathbb{Z} / p^{j} \mathbb{Z}\right]$.

(2) For $0<q<n$,

$$
\begin{gathered}
K(n)_{*}\left(K\left(\mathbb{Z} / p^{j} \mathbb{Z}, q\right)\right) \cong \bigotimes_{I} P_{K(n)_{*}}\left[a_{I}, \rho(I)\right], \\
I=\left(i_{1}, \ldots, i_{q}\right), n(j-1)<i_{1}<\cdots<i_{q}<n_{j},
\end{gathered}
$$

where $\rho(I)$ is determined by the fact that $K(n)_{*}\left(K\left(\mathbb{Z} / p^{j} \mathbb{Z}, \bullet\right)\right)$ is the free Hopf ring over $K(n)_{*}\left[\mathbb{Z} / p^{j} \mathbb{Z}\right]$ on the Hopf algebra $K(n)_{*}\left(K\left(\mathbb{Z} / p^{j} \mathbb{Z}, 1\right)\right)$.

(3) When $q=n$,

$$
\begin{gathered}
K(n)_{*}\left(K\left(\mathbb{Z} / p^{j} \mathbb{Z}, n\right)\right) \cong \bigotimes_{I} K(n)_{*}\left[a_{I}\right] /\left(a_{I}^{* p}+(-1)^{n} v_{n}^{c(I)} a_{I}\right) \\
c(I)=p^{n(j-1)}-(p-1)\left(p^{n(j-2)}+p^{n(j-3)}+\cdots+p^{n k}\right), \\
I=(n k, n(j-1)+1, \ldots, n j-1), \quad 0 \leq k<j .
\end{gathered}
$$

(4) $K(n)_{*}\left(K\left(\mathbb{Z} / p^{j} \mathbb{Z}, q\right)\right) \cong K(n)_{*}$ for $q>n$.

We are interested in the dimension of $K(n)_{*}(K(\pi, q))$ as vector spaces over $K(n)_{*}$. For $\pi=\mathbb{Z}$, there is an isomorphism

$$
\lim _{j} K(n)_{*}\left(K\left(\mathbb{Z} / p^{j} \mathbb{Z}, q\right)\right) \cong K(n)_{*}(K(\mathbb{Z}, q+1)),
$$

and so it follows that $K(n)_{*}(K(\mathbb{Z}, q))$ is infinite-dimensional over $K(n)_{*}$. We therefore restrict our attention to the case where $\pi$ is finite. 
Proposition 2.3. $\log _{p} \operatorname{dim}_{K(n) *} K(n)_{*}\left(K\left(\mathbb{Z} / p^{j} \mathbb{Z}, q\right)\right)=j\left(\begin{array}{l}n \\ q\end{array}\right)$.

Proof. We begin with $\pi=\mathbb{Z} / p \mathbb{Z}$, where there is a more accessible accounting of the algebra structure for the Morava $K$-theory. Following [10], we have

$$
K(n)_{*}(K(\mathbb{Z} / p \mathbb{Z}, q)) \cong \bigotimes_{I} P_{K(n)_{*}}\left[a_{I}, \rho(I)\right],
$$

where for $I=\left(i_{1}, \ldots, i_{q}\right), \rho(I)=1+\max \{0\} \cup\left\{s+1 \mid i_{q-s}=n-1-s\right\}$.

For convenience, we denote $\log _{p} \operatorname{dim}_{K(n) *} K(n)_{*}\left(K\left(\mathbb{Z}_{i}^{\prime} p^{j} \mathbb{Z}, q\right)\right)$ by $x_{n, q}^{j}$. From the tensor product representation of $K(n)_{*}(K(\mathbb{Z} / p \mathbb{Z}, q))$, it follows immediately that $x_{n, q}^{1}=\sum_{I} \rho(I)$. We consider the case $0<q<n$. Then $1 \leq \rho(I) \leq q+1$. Let

$$
x_{n, q}^{1}=\sum_{k=1}^{q+1} k \cdot \# k
$$

where $\# k=\#\{I \mid \rho(I)=k\}$. Counting down from $q+1$, we see that $\rho(I)=q-k$ when

$$
I=\left(i_{1}, \ldots, i_{2}, \ldots, i_{k+1}, n-q+k+1, \ldots, n-1\right) .
$$

There are $\left(\begin{array}{c}n-q+k-1 \\ k+1\end{array}\right)$ choices for the undetermined $i_{l}$. Thus

$$
x_{n, q}^{1}=\sum_{k=1}^{q+1}(q-k)\left(\begin{array}{c}
n-q+k-1 \\
k+1
\end{array}\right)=\sum_{k=0}^{q}(q+1-k)\left(\begin{array}{c}
n-q-2+k \\
k
\end{array}\right) .
$$

This can be rewritten in the form

$$
x_{n, q}^{1}=\sum_{k=0}^{q}\left(\begin{array}{c}
q+k-1 \\
1
\end{array}\right)\left(\begin{array}{c}
n-q-2+k \\
n-q-2
\end{array}\right) .
$$

Now we appeal to a combinatorial identity that is proved by counting subsets in a clever fashion

$$
\text { Combinatorial fact: }\left(\begin{array}{l}
N \\
Q
\end{array}\right)=\sum_{L=d}^{N-Q+d}\left(\begin{array}{l}
L-1 \\
d-1
\end{array}\right)\left(\begin{array}{l}
N-L \\
Q-d
\end{array}\right) \text {. }
$$

In the formula for $x_{n, q}^{1}$, by letting $d=n-q-1, L=n-q-1+k$, we get $N=n$ and $Q=n-q$. Substituting into the sum, we have

$$
\begin{aligned}
x_{n, q}^{1} & =\sum_{k=0}^{q}\left(\begin{array}{c}
q+k-1 \\
1
\end{array}\right)\left(\begin{array}{c}
n-q-2+k \\
n-q-2
\end{array}\right) \\
& =\sum_{L=n-q-1}^{n-1}\left(\begin{array}{c}
L-1 \\
n-q-2
\end{array}\right)\left(\begin{array}{c}
n-L \\
1
\end{array}\right)=\left(\begin{array}{c}
n \\
n-q
\end{array}\right)=\left(\begin{array}{l}
n \\
q
\end{array}\right) .
\end{aligned}
$$

The argument above holds for $0<q<n$. In the case of $q=n$, we are computing the dimension over $k$, a field, of $k[x] /\left(x^{p}-\alpha k\right)$ where $\alpha$ is a nonzero element of $k$. This is clearly equal to $p$ so that

$$
\log _{p} \operatorname{dim}_{K(n)_{*}} K(n)_{*}(K(\mathbb{Z} / p \mathbb{Z}, n))=1=\left(\begin{array}{l}
n \\
n
\end{array}\right) .
$$

Finally, when $n<q, \log _{p} \operatorname{dim}_{K(n)_{*}} K(n)_{*}(K(\mathbb{Z} / p \mathbb{Z}, q))=0$, which is consistent with $\left(\begin{array}{l}n \\ q\end{array}\right)$. 
Next we consider the case $j>1$. According to [10], we know that the vector spaces $K(n)_{*}\left(K\left(\mathbb{Z} / p^{j} \mathbb{Z}, q\right)\right)$ are generated by products of the form $a_{\left(i_{1}\right)} \circ \cdots \circ$ $a_{\left(i_{q}\right)}$, where the $a_{\left(i_{j}\right)}$ are generators in $K(n)_{*}\left(K\left(\mathbb{Z} / p^{j} \mathbb{Z}, 1\right)\right)$. Since $a_{(i)} \circ a_{(i)}=0$, there are $\left(\begin{array}{c}n j \\ q\end{array}\right)$ such products to consider. However, the following relations hold among these generators:

(1) $a_{(i)} \circ a_{(k)}=-a_{(k)} \circ a_{(i)}$.

(2) $a_{(i)} \circ a_{(k)}=0$ when $i<k$ and $k<n(j-1)$.

(3) $a_{(i)} \circ a_{(k)}=a_{(i-n)} \circ a_{(k+n)}$ when $n \leq i$ and $k<n(j-1)$.

(4) $a_{(i)}^{* p}=v_{n}^{m} a_{(i+1-n)}$ for some $m \in \mathbb{Z}$, and $\left(a_{(i)} \circ a_{(k)}\right)^{* p}=a_{(i+1)} \circ a_{(k)}^{* p}$ for $i<n j-1$.

Since $\operatorname{dim}_{K(n) *} K(n)_{*}(X)=\operatorname{dim}_{\mathbb{F}_{p}} \overline{K(n)}_{*}(X)$, we can examine these relations in $\overline{K(n)}_{*}\left(K\left(\mathbb{Z} / p^{j} \mathbb{Z}, q\right)\right)$. For $q<n$, one finds that the generators for $\overline{K(n)}_{*}\left(K\left(\mathbb{Z} / p^{j} \mathbb{Z}, q\right)\right)$ are indexed over the set

$$
\begin{array}{r}
B=\left\{I \mid 0 \leq i_{1}<i_{2}<\cdots<i_{q}<n j, \text { with } n(j-\mathrm{i})<i_{2}\right. \\
\text { and } \left.0 \leq \bar{i}_{1}<\bar{i}_{2}<\cdots<\bar{i}_{q}<n\right\},
\end{array}
$$

where $\bar{i}_{k}$ is the remainder on division of $i_{k}$ by $n$.

To see that all of these are independent in $\overline{K(n)}_{*}\left(K\left(\mathbb{Z} / p^{j} \mathbb{Z}, q\right)\right)$ requires repeated applications of the facts above. The values of the $\rho(I)$ in Theorem 2.2 result from such a computation, as well as the fact that the dimension we are seeking has $p$-logarithm equal to the number of elements in $B[10$, Theorem 11.1(b)]). Now we show that $B$ has the desired cardinality.

Pick $\bar{i}_{2}=l$, with $1 \leq l \leq n-q+1$. To complete the sequence with $i_{3}$ to $i_{q}$ we have $\left(\begin{array}{c}n-1-l \\ q-2\end{array}\right)$ choices. There are now $l$ choices for $\bar{i}_{1}$, and to each choice there are $j$ representatives among $0 \leq i_{1}<i_{2}$. Therefore,

$$
\# B=\sum_{l=1}^{n-q+1} j l\left(\begin{array}{c}
n-1-l \\
q-2
\end{array}\right)=j \sum_{L=2}^{n-q+2}\left(\begin{array}{l}
L-1 \\
2-1
\end{array}\right)\left(\begin{array}{c}
n-L \\
q-2
\end{array}\right) .
$$

Applying the combinatorial fact, we see that $\# B=j\left(\begin{array}{l}n \\ q\end{array}\right)$.

When $q=n$, the explicit expression for $K(n)_{*}\left(K\left(\mathbb{Z} / p^{j} \mathbb{Z}, n\right)\right)$ in the theorem above gives $x_{n, n}^{j}=j$, which is the same as $j\left(\begin{array}{l}n \\ n\end{array}\right)$. For $q>n, x_{n, q}^{j}=0=j\left(\begin{array}{l}n \\ q\end{array}\right)$.

Theorem 2.4. Let $\pi$ be a finite group and $p$ be an odd prime. Then for $q \geq 1$,

$$
\chi\left(K(n)_{*}(\mathscr{L} K(\pi, q))\right)=\chi\left(K(n+1)_{*}(K(\pi, q))\right) .
$$

Proof. The case $q=1$ was shown in [7]. Therefore, we may assume that $q>1$ and $\pi$ is abelian. Since $K(n)_{*}(-)$ is a $p$-local theory, we need only consider the case when $\pi=\mathbb{Z} / p^{j} \mathbb{Z}$.

The $H$-space structure on $K(\pi, q)$ yields a product decomposition,

$$
\mathscr{L} K(\pi, q) \simeq K(\pi, q) \times \Omega K(\pi, q) \simeq K(\pi, q) \times K(\pi, q-1) .
$$

Applying $K(n)_{*}$, we get

$$
K(n)_{*}(\mathscr{L} K(\pi, q)) \cong K(n)_{*}(K(\pi, q)) \otimes_{K(n)_{*}} K(n)_{*}(K(\pi, q-1)) .
$$

Since $K(n)_{*}(K(\pi, q))$ has nothing in odd degrees, its dimension as a vector space over $K(n)_{*}$ is equal to its Euler characteristic. Therefore, it is enough to show that

$\log _{p} \operatorname{dim}_{K(n) *} K(n)_{*}\left(\mathscr{L} K\left(\mathbb{Z} / p^{j} \mathbb{Z}, q\right)\right)=\log _{p} \operatorname{dim}_{K(n+1)_{*}} K(n+1)_{*}\left(K\left(\mathbb{Z} / p^{j} \mathbb{Z}, q\right)\right)$. 
But from Proposition 2.3 and the remarks above, we have that

$$
\log _{p} \operatorname{dim}_{K(n) *} K(n)_{*}\left(\mathscr{L} K\left(\mathbb{Z} / p^{j} \mathbb{Z}, q\right)\right)=j\left(\left(\begin{array}{c}
n \\
q
\end{array}\right)+\left(\begin{array}{c}
n \\
q-1
\end{array}\right)\right) .
$$

Since

$$
\left(\begin{array}{l}
n \\
q
\end{array}\right)+\left(\begin{array}{c}
n \\
q-1
\end{array}\right)=\left(\begin{array}{c}
n+1 \\
q
\end{array}\right)
$$

by Pascal's triangle, the result follows.

\section{The Morava $K$-Theory of $\mathscr{L} \Sigma X$}

One could try to generalize Theorem 2.4 to other spaces by using the Postnikov tower, which behaves well with respect to the free loop functor. At the moment, the machinery for computing the Morava $K$-theory of a pullback from the given data is lacking. For a general space $Z$, one can utilize Goodwillie's model of the free loop space [6] to obtain a spectral sequence abutting to $K(n)_{*}(\mathscr{L} Z)$ with $E_{2}$-term given by the Hochschild homology of $K(n)_{*}(\Omega Z)$. However, as the example in [5] shows, $K(n)_{*}(Z)$ may be trivial while $K(n)_{*}(\Omega Z)$ is not.

One notable exception to this is when $Z$ is a suspension $\Sigma X$. In this case, there is an explicit combinatorial model for $\mathscr{L} \Sigma X$, analogous to the James model. This is described in [4], and we now show that the argument given there applies to compute $K(n)_{*}(\mathscr{L} \Sigma X)$ in terms of $K(n)_{*}(X)$. Recall

Theorem 3.1 [4]. Let $C_{n}$ denote the cyclic group of order $n$. Let $X$ be a pointed topological space with basepoint $*$. Define the space

$$
R(X)=\bigvee_{n \geq 1} S_{+}^{1} \wedge_{C_{n}} X^{\wedge n}
$$

Then the spaces $R(X)$ and $\mathscr{L} \Sigma X$ are stably homotopy equivalent.

Here $Y_{+}$denotes the space $Y$ with a disjoint basepoint adjoined, $X^{\wedge n}=$ $X \wedge \cdots \wedge X$ ( $n$ times), and $C_{n}$ acts on $S^{1}$ as multiplication by $e^{2 \pi i / n}$.

For any generalised homology theory $h_{*}$ which satisfies the wedge axiom, we then have

$$
h_{*}(\mathscr{L} \Sigma X) \cong \bigoplus_{n \geq 1} h_{*}\left(S_{+}^{1} \wedge_{C_{n}} X^{\wedge n}\right)
$$

The space $S_{+}^{1} \wedge_{C_{n}} X^{\wedge n}$ is obtained from $S^{1} \times{ }_{C_{n}} X^{n}$ by identifying to a point the subspace $S^{1} \times_{C_{n}} F W_{n}(X)$, where $F W_{n}(X)$ is the subspace of $X^{n}$ of $n$ tuples with some entry $x_{i}=*$ (the fat wedge). Now $S^{1} \times{ }_{C_{n}} X^{n}$ is the total space of a fibration

$$
X^{n} \hookrightarrow X^{1} \times_{C_{n}} X^{n} \rightarrow S^{1} \times_{C_{n}} * \cong S^{1} .
$$

Applying the Atiyah-Hirzebruch spectral sequence, we get an $E^{2}$-term

$$
E_{*, *}^{2} \cong H_{*}\left(S^{1} ; h_{*}\left(X^{n}\right)\right) \text {. }
$$

When $h_{*}$ has a Künneth isomorphism, we can write $E^{2}$ as

$$
E_{*, *}^{2} \cong H_{*}\left(S^{1} ; h_{*}(X)^{\otimes n}\right) \text {, }
$$

where the tensor product is taken over $h_{*}(p t)$. For dimensional reasons, this spectral sequence collapses. 
If $G$ is a graded vector space over a field $k$, then, as we have in the case above, $H_{*}\left(S^{1} ; G^{\otimes n}\right)$ denotes the twisted homology of $S^{1}$ with twisted coefficients determined by the action of $\pi_{1} S^{1} \cong \mathbb{Z}$ induced by the projection of $\mathbb{Z}$ onto $\mathbb{Z} / n \mathbb{Z}$ with $t_{n}$ the image of 1 . The action is given by

$$
t_{n}\left(g_{1} \otimes \cdots \otimes g_{n}\right)=(-1)^{n-1}\left(g_{n} \otimes g_{1} \otimes \cdots \otimes g_{n-1}\right)
$$

This group homology has been computed classically [12].

Proposition 3.2. $H_{q}\left(S^{1} ; h_{*}(X)^{\otimes n}\right)$ is given by

$$
\begin{cases}h_{*}(X)^{\otimes n} /\left(1-t_{n}\right), & \text { if } q=0 \\ \left(h_{*}(X)^{\otimes n}\right)^{t_{n}}, & \text { if } q=1 \\ \{0\}, & \text { if } q \geq 2 .\end{cases}
$$

As discussed in [3] this leads to an expression for $h_{*}\left(S_{+}^{1} \wedge_{C_{n}} X^{\wedge n}\right)$. In particular, there is the cofibration sequence

$$
S^{1} \times_{C_{n}} F W_{n}(X) \hookrightarrow S^{1} \times_{C_{n}} X^{n} \rightarrow S_{+}^{1} \wedge_{C_{n}} X^{\wedge n} .
$$

Therefore,

$$
h_{*}\left(S_{+}^{1} \wedge_{C_{n}} X^{\wedge n}\right) \cong H_{*}\left(S^{1} ; h_{*}(X, *)^{\otimes n}\right) .
$$

Writing $\tilde{h}_{*}(X)$ for the reduced homology functor $h_{*}(X, *)$ and applying 3.2, we have

$$
h_{*}\left(S_{+}^{1} \wedge_{C_{n}} X^{\wedge n}\right)=\left[\tilde{h}_{*}(X)^{\otimes n} /\left(1-t_{n}\right)\right] \oplus s\left(\tilde{h}_{*}(X)^{\otimes n}\right)^{t_{n}},
$$

where $s$ is the suspension functor on graded modules $\left((s M)_{n}=M_{n-1}\right)$.

Putting all this together, we have the isomorphism

$$
h_{*}(\mathscr{L} \Sigma X) \cong \bigoplus_{j \geq 1}\left[\tilde{h}_{*}(X)^{\otimes j} /\left(1-t_{j}\right)\right] \oplus s\left(\tilde{h}_{*}(X)^{\otimes j}\right)^{t_{j}}
$$

Of course, the Morava $K$-theories for odd primes satisfy the assumptions required of $h_{*}$ to obtain these results. Since $K(n)_{*}$ is a field, we can further write that

$$
K(n)_{*}(\mathscr{L} \Sigma X) \cong H H_{*}\left(T\left(K(n)_{*}(X, *)\right)\right),
$$

where $\mathrm{HH}_{*}$ is the Hochshchild homology of a graded algebra, and $T(-)$ denotes the tensor algebra functor over $K(n)_{*}$. Finally, we remark that the argument still goes through if $h_{*}(X)$ is free but $h_{*}$ itself does not have a Künneth isomorphism. This is the case for instance, if one wanted to compute $K^{*}\left(\mathscr{L} S^{n}\right)$.

\section{ACKNOWLEDGMENT}

We wish to acknowledge helpful conversations with Ralph Cohen and Doug Ravenel, and we thank MSRI for providing support for this research.

\section{REFERENCES}

1. J.-L. Brylinski, Representations of loop groups, Dirac operators on loop space, and modular forms, Topology 29 (1990), 461-480.

2. E. H. Brown and F. P. Peterson, A spectrum whose $\mathbb{Z}_{p}$-cohomology is the algebra of reduced pth powers, Topology 5 (1966), 149-154.

3. G. E. Carlsson and R. L. Cohen, The cyclic groups and the free loop space, Comment. Math. Helv. 62 (1987), 423-449. 
4. R. L. Cohen, $A$ model for the free loop space of a suspension, Algebraic Topology (Proceedings, Seattle, 1985), Lecture Notes in Math., vol. 1286, Springer-Verlag, New York, 1987, pp. 193-207.

5. T. Ghazal, A new example in the $K$-theory of loop spaces, Proc. Amer. Math. Soc. 107 (1989), 855-856.

6. T. Goodwillie, Cyclic homology, derivations, and the free loop space, Topology 24 (1985), 187-215.

7. M. J. Hopkins, N. J. Kuhn, and D. C. Ravenel, Generalized group characters and complex oriented cohomology theories (to appear).

8. D. C. Johnson and W. S. Wilson, BP operations and Morava's extraordinary $K$-theories, Math. Z. 144 (1975), 55-75.

9. H. Miller, The elliptic character and the Witten genus, Algebraic Topology (Proc. of the Internat. Conf., Evanston, 1988), Contemp. Math. 96 (1989), 281-289.

10. D. C. Ravenel and W. S. Wilson, The Morava K-theory of Eilenberg-Mac Lane spaces and the Conner-Floyd conjecture, Amer. J. Math. 102 (1980), 691-748.

11. G. Segal, Elliptic cohomology, Astérique 161-162 (1988), 187-201.

12. N. Steenrod and D. B. A. Epstein, Cohomology operations, Princeton Univ. Press, Princeton, NJ, 1962.

13. E. Witten, Elliptic genera and quantum field theory, Comm. Math. Phys. 109 (1987), 525536.

14. _ The index of the Dirac operator in loop space, Elliptic Curves and Modular Forms in Algebraic Topology (Proceedings, Princeton, 1986), Lecture Notes in Math., vol. 1326 Springer-Verlag, New York, 1988, pp. 161-181.

Department of Mathematics, Vassar College, Poughreepsie, New York 12601

Department of Mathematics, Princeton University, Princeton, New Jersey 08544 